THE Astrophysical JourNal, $227:$ :L5-L.58, 1979 January 15

(c) 1979. The American Astronomical Society. All rights reserved. Printed in U.S.A.

\title{
ALIGNMENT OF THE OPTICAL POLARIZATION WITH THE RADIO STRUCTURE OF QSOS
}

\author{
H. S. Stockman and J. R. P. Anged \\ Steward Observatory, University of Arizona
}

AND

\author{
G. K. MILEY
}

Sterrewacht, Leiden, The Netherlands; and Lick Observatory, University of California at Santa Cruz Received 1978 September 19; accepted 1978 October 2

\begin{abstract}
Using the results of the recently completed linear polarization survey of bright QSOs, we have compared the position angle of linear polarization with the orientation of associated double radio sources. Of the 24 objects for which both position angles are known to better than $15^{\circ}, 20$ have the two angles agreeing to within $30^{\circ}$. The probability that such alignment occurs by chance is less than $10^{-5}$. On physical grounds, the common orientation of the optical linear polarization and the extended radio lobes indicates a long-term memory of direction in these QSOs, similar to that found in nearby radio galaxies by VLBI observations. The reason for the alignment of the rather small polarization, typically $1 \%$, is unknown. We discuss several possible polarizing mechanisms, including synchrotron radiation near the central source and scattering within the surrounding host galaxy. Measurements of the emission-line polarizations and variability studies of the continuum polarization should be capable of distinguishing between the various sources of polarization and thus may indicate the nature of the observed alignment.
\end{abstract}

Subject headings: polarization - quasars

I. RELATION OF RADIO STRUCTURE TO RECENT OPTICAL POLARIZATION DATA

Studies of the morphologies of some double radio galaxies from scales of parsecs to megaparsecs indicate that the lobes are produced by a process in the nuclei that both is highly directional and can maintain its direction over periods in excess of $10^{8}$ years (Fomalont and Miley 1975; Readhead, Cohen, and Blandford 1978). Here we show that a comparison of the optical polarization angle in QSOs with the orientation of their radio structure provides further evidence for the existence of fundamental axes in double radio sources.

A recent polarization survey of bright QSOs (Stockman and Angel 1978; Stockman 1978) has produced linear polarization measures for approximately 130 QSOs with typical accuracies of a few tenths of a percent. The basic results show that, except for a small number of violently variable QSOs whose polarimetric properties resemble those of the BL Lacertae objects, most QSOs are weakly polarized at the $1 \%$ level or less. Of the objects studied, 24 are quasars with extended radio emission larger than a kiloparsec, for which position angles of both optical polarization and radio structure have been measured to better than $15^{\circ}$. These are listed in Table 1 together with information on their radio structures and optical polarization. For a few QSOs, the optical polarization data have not been published elsewhere. When not specified in the literature, uncertainties in the structural parameters have been estimated. For radio sources which were sufficiently resolved, the morphological type is also indicated in Table 1. In most cases, the sources have the classic symmetric (S) double-lobed structure characteristic of high-luminosity radio sources with steep spectra. The radio emission in 3C 273 and 3C 345, however, is dominated by the flat-spectrum nuclear components and the associated extended structure appears one-sided and asymmetric (A).

Figure 1 shows a histogram of $|\Delta \phi|$, the modulus of the difference between the position angles of radio structure and optical polarization. The distribution is clearly nonrandom. For a random distribution we would expect eight of the 24 objects to have $|\Delta \phi|<$ $30^{\circ}$, whereas we find 20 . This is improbable at a level $<1$ in $10^{5}$. Moreover, the four points above the dotted line in the histogram were obtained after our correlation was originally discovered. All have $|\Delta \phi|<13^{\circ}$, increasing our confidence in the reality of the relation.

Figure 1 indicates that the optical polarization is aligned almost but not exactly along the radio source. The median $|\Delta \phi|$ of $\sim 20^{\circ}$ is larger than one would expect from the formal uncertainties in the position angles if the angles were exactly aligned. However, interstellar polarization of a few tenths of a percent is expected from dust clouds at high galactic latitude. This will change the direction of optical polarization by as much as $10^{\circ}$ on average and could easily broaden the histogram by the observed amount. Further data are needed to establish whether the optical polarizations are indeed exactly aligned along the radio source or whether they are slightly offset. 


\section{DISCUSSION}

An elementary conclusion to be drawn from the striking alignment of polarization and lobe axes is that , the long-term memory of direction established for some in radio galaxies is also a feature of quasars with similar Iin radio morphology. The radio lobes which are located typically $\sim 300 \mathrm{kpc}$ from the QSOs must have originated in the QSO nucleus more than at least $10^{6}$ years before the optical emission. The common remembered axis of polarization and lobes may simply be that of the rotation of a host galaxy or of a central massive object not necessarily aligned with the galaxy. In the following discussion we consider possible origins for the polarization and its alignment.

\section{a) Intrinsic Polarization}

The most basic question is whether the polarization is an intrinsic property of the emission process or whether it arises due to scattering of the emitted radiation. In some violently variable QSOs and BL Lacertae objects the optical polarizations are so large and highly variable that the optical continuum emission must be produced by an intrinsically polarized mecha- nism, presumably synchrotron radiation. Can we attribute the weaker polarization seen in the more commonly observed quiescent QSOs to the same mechanism? Some similarity between the properties of weak and strong polarizations suggests that both may indeed have a common origin. In highly polarized objects the direction of polarization often varies over all angles but about half do have a preferred direction $\left( \pm 20^{\circ}\right)$ over a period of at least years (Angel et al. 1978). In the case of $3 \mathrm{C} 345$, a violently variable QSO, measurements over several years indicate that, while the position angle of this highly polarized object wanders considerably, on average it appears to line up roughly with the milli-arcsec radio structure (Kinman 1977).

If the weak and strong polarizations are both intrinsic, they may reflect the magnetic field structure at the primary energy source of the QSO $\left(R \sim 10^{15} \mathrm{~cm}\right.$ by variability studies). In the quiescent stage of the quasar the polarization may be determined by the average orientation of the magnetic field over the entire region. The alignment of the weak polarization would then indicate that the field, although far from uniform, has a residual component perpendicular to

TABLE 1

\begin{tabular}{|c|c|c|c|c|c|c|c|c|c|}
\hline \multirow[t]{2}{*}{ QSO } & \multirow[t]{2}{*}{ Alt. Names } & \multicolumn{5}{|c|}{ Radio Structure } & \multicolumn{2}{|c|}{ Optical Polarization } & \multirow{2}{*}{$\frac{\Delta \phi}{(\mathrm{deg})}$} \\
\hline & & Type & $\begin{array}{l}\text { Largest } \\
\text { angular } \\
\text { size } \\
\text { (arc sec) }\end{array}$ & $\begin{array}{r}\text { p.a. } \\
\text { (deg) }\end{array}$ & & $\operatorname{Ref}$ & Percentage & $\begin{array}{c}\text { p.a. } \\
(\mathrm{deg})\end{array}$ & \\
\hline $0003+158$ & 4C 15.01 , PHL 658 & $\mathrm{~s}$ & $31.5+1.8$ & $115+$ & 6 & 1 & $0.68+0.20$ & $100+$ & $+15+11$ \\
\hline $0130+242$ & 4C 24.02, PKS & $\mathrm{s}$ & $54.6 \pm 1.0$ & $93 \pm$ & 3 & 1 & $1.70 \pm 0.52$ & $110 \pm 9$ & $-17 \pm 9$ \\
\hline $0159-117$ & 3C 57 , PKS & A & $16.5 \pm 2$ & $7 \pm$ & 2 & 2 & $0.64 \pm 0.30$ & $1 \pm 13$ & $+6 \pm 13$ \\
\hline $0214+108$ & 4C 10.06 , PKS & $S$ & $119 \mp 2$ & $75 \pm$ & 3 & 1 & $1.27 \pm 0.24$ & $102 \mp 5$ & $-27 \pm 6$ \\
\hline $0349-146$ & 3C 95 , PKS & $\mathrm{s}$ & $114 \pm 4$ & $165.5 \pm$ & 0.7 & 1 & $1.33 \pm 0.51$ & $160 \pm 11$ & $+6 \pm 11$ \\
\hline $0350-073$ & 3C 94, PKS & $\mathrm{s}$ & $41.5 \pm 1.0$ & $90 \mp$ & 11 & 1 & $1.20 \pm 0.27$ & $24 \pm 6$ & $+66 \pm 13$ \\
\hline $0405-123$ & PKS & & $19 \pm 5$ & $3 \pm$ & 10 & 1 & $0.83 \pm 0.16$ & $136 \pm$ & $+47 \pm 11$ \\
\hline $0742+318$ & 4C $31.30, \mathrm{~B} 2$ & $\mathrm{~s}$ & $115 \pm 2$ & $125 \mp$ & 2 & 3 & $0.58 \mp 0.18$ & $5 \mp 9$ & $-60 \mp 9$ \\
\hline $1043-090$ & 3C 246 , PKS & S & $83 \mp 3$ & $122 \mp$ & 4 & 1 & $0.85 \mp 0.30$ & $96 \mp 10$ & $+26 \mp 11$ \\
\hline $1100+772$ & 3C 249.1, 4C 77.09 & $\mathrm{~s}$ & $48 \mp 1$ & $90 \bar{t}$ & 1 & 1 & $0,71 \pm 0,22$ & $76 \mp 8$ & $+14 \pm 8$ \\
\hline $1104+167$ & 4C 16.30 & & $6.8 \pm 1$ & $129 \pm$ & 10 & 4 & $0.56 \pm 0.21$ & $160 \mp 11$ & $-31 \pm 15$ \\
\hline $1128+315$ & B2 & & $14 \pm 3$ & $178 \pm$ & 10 & 3 & $0.95 \pm 0.33$ & $172 \pm 10$ & $+6 \pm 14$ \\
\hline $1208+322$ & B2 & $\mathrm{s}$ & $75 \pm 2$ & $3 \pm$ & 5 & 3 & $1.03 \pm 0.24$ & $26 \pm 7$ & $-23 \pm 9$ \\
\hline $1226+023$ & $3 \mathrm{C} 273,4 \mathrm{C} 02.32$ & A & $20 \pm 1$ & $45 \pm$ & 3 & 5 & $0.21 \pm 0.04$ & $52 \pm$ & $-7 \pm$ \\
\hline $1425+267$ & B2, Ton 202 & $\mathrm{~s}$ & $218 \mp 2$ & $56 \mp$ & 3 & 1 & $1.41 \mp 0.23$ & $74 \mp$ & $-18 \overline{ \pm}$ \\
\hline $1512+370$ & $4 \mathrm{C} 37.43, \mathrm{~B} 2$ & $\mathrm{~s}$ & $55 \pm 1$ & $110 \pm$ & 2 & 1 & $1.10 \pm 0.23$ & $109 \mp$ & $+1 \pm$ \\
\hline $1545+210$ & 3C $323.1,4 \mathrm{C} 21.45, \mathrm{PKS}$ & $\mathrm{s}$ & $69 \pm 3$ & $21 \mp$ & 1 & 1 & $1.65 \mp 0.24$ & $16 \mp$ & $+5 \bar{t}$ \\
\hline $1618+177$ & $3 \mathrm{C} 334,4 \mathrm{C} 17.68$, PKS & $\mathrm{s}$ & $49 \mp 2$ & $118 \mp$ & 3 & 1 & $0.81 \pm 0.42$ & $95 \mp 15$ & $+23 \pm 15$ \\
\hline $1634+269$ & $3 \mathrm{C} 342,4 \mathrm{C} 26.49, \mathrm{~B} 2$ & $\mathrm{~s}$ & $39 \pm 1$ & $107 \pm$ & 3 & 1 & $2,05 \pm 0,76$ & $120 \mp 11$ & $-13 \pm 11$ \\
\hline $1622+238$ & $3 \mathrm{C} 336,4 \mathrm{C} 23.43$ & $\mathrm{~s}$ & $22 \pm 2$ & $30 \div$ & 4 & 1 & $2.98 \pm 1.37$ & $51 \mp 13$ & $-21 \pm 14$ \\
\hline $1641+399$ & $3 \mathrm{C} 345,4 \mathrm{C} 39.48$ & A & $2.1 \pm 0.1$ & $149 \pm$ & 6 & 6 & $3.57 \pm 0.19$ & $173 \pm 2$ & $-24 \pm 6$ \\
\hline $1721+343$ & $4 C 34.47$ & $\mathrm{~s}$ & $220 \pm 6$ & $162 \pm$ & 1 & 7 & $1.14 \pm 0.25$ & $145 \pm 7$ & $+17 \pm 7$ \\
\hline $2120+168$ & $3 \mathrm{C} 432,4 \mathrm{C} 16.72$, PKS & $\mathrm{S}$ & $12.9 \pm 0.8$ & $130 \pm$ & 5 & 8 & $1.98 \pm 0.93$ & $135 \pm 13$ & $-5 \pm 13$ \\
\hline $2308+098$ & $4 C 09.72$ & $S$ & $101 \mp 5$ & $147 \pm$ & 2 & 1 & $1.08 \pm 0.24$ & $121 \pm 6$ & $+26 \pm 6$ \\
\hline
\end{tabular}

1. Miley and Hartsuijker (1978).

2. Wilkinson et al. (1974).

3. Fanti et al. (1977).

4. Wardle and Miley (1974).
5. Anderson and Donaldson (1967).

6. Davis et al. (1977).

7. Conway et al. (1977).

8. Hogg (1969). 


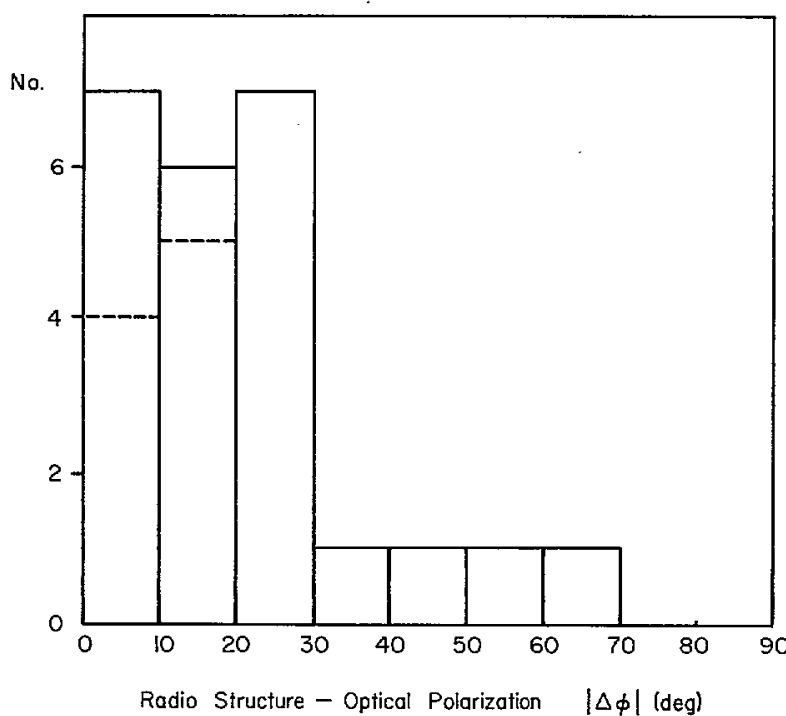

FIg. 1.-Histogram of the absolute difference between the angles of radio elongation and optical polarization, $|\Delta \phi|$, from the data in Table 1. The parts of the histogram above the dotted line were measured after the relation between the two angles was discovered.

the radio ejection axis. Outbursts localized at different places within the magnetic field region would explain the extreme variability in both polarization percentage and direction often seen in strongly polarized QSOs. Magnetic fields associated with massive objects are features of several radio source models (e.g., Sanders 1974; Lovelace 1976; Blandford 1976).

Another possibility is that polarized optical synchrotron emission is produced in jets on a larger scale than the central source discussed above. The optical continuum source could have the same type of morphology as a typical radio source, with a pair of lobes elongated along the fundamental radio axis. VLBI measurements have shown that the brightness distributions of the central radio cores are in general similar to that of the extended double sources on a scale $10^{6}$ times smaller. One might expect the magnetic field directions in these small cores to also resemble those found in extended radio sources, with the field directed along the radio axis in the inner part of the lobes, but perpendicular to this axis at the outer edges of the source (e.g., Miley 1976). Analogous behavior for the optical continuum could explain the observed alignment, provided the outer edges of the optical source (perpendicular fields) were brighter. Perhaps the optical emission is entirely produced where the plasmoids responsible for the radio core emission interact with the host galaxy. On a still larger scale, an extended jet with a size of tens of kiloparsecs, like those in M87 and 3C 273, would typically be included in our $4^{\prime \prime}$ aperture and could be the site of the observed polarization. We do not find this a very attractive explanation, though, as the net polarizations and luminosities of the known jets are small (Schmidt, Peterson, and Beaver 1978).

\section{b) Polarization by Scattering}

The observed polarization may not be produced with the optical emission but may arise in the surrounding medium, either from scattering by a surrounding nonspherical cloud or from transmission through aligned grains. Dust scattering is responsible for much of the polarization found in the nuclei of both Seyfert 1 and Seyfert 2 galaxies (Stockman, Angel, and Beaver 1976). For example, a study of emission-line polarizations has shown that the polarizing dust lies between the permitted-line and forbidden-line-formation regions in NGC 1068 (Angel et al. 1976). Polarization by aligned grains along the line of sight, the mechanism responsible for interstellar polarization in our own Galaxy, is not a very plausible origin for QSO polarization. This polarization is produced by small differences in extinction, depending on the orientation of the grains, and would require a large total extinction, incompatible with the observed ultraviolet spectra. We also note that alignment by a magnetic field like that of our own Galaxy would result in polarization perpendicular to the minor axis. Little or no extinction is required if the polarization originates in a surrounding, asymmetric scattering cloud of electrons or dust. For an optically thin cloud, the polarization will be parallel to the minor axis, while for an optically thick cloud, the polarization will be along the major axis (Angel 1969). If, as seems likely, the fundamental radio axis is rotational in origin, we might expect any gas surrounding the QSO to have its minor axis directed along the radio axis. Thus scattering by an optically thin cloud is a plausible explanation of our correlation (Rees 1978). The required optical depth depends on the degree of asymmetry. But since the observed polarization is small $(1 \%)$ and scattered photons are highly polarized, the range of optical depths for reasonable asymmetry is $\tau \sim 0.02-0.10$. Clouds of this optical depth would be difficult to detect spectroscopically or photometrically. For nearby QSOs, however, galactic scattering clouds may be resolvable under conditions of good seeing or by polarimetric mapping (see also Jura 1978).

Polarization arising outside the emission-line regions either from scattering or from transmission through aligned grains will affect the emission lines as well as the continuum. Therefore accurate spectropolarimetry of several aligned QSOs in our sample should be able to place the source of polarization and the apparent alignment with respect to the broad-line emission region. Furthermore, if the scatterers are dust, we would expect to see a strong wavelength dependence of polarization, similar to that seen in the nuclei of Seyfert galaxies (Maza, Martin, and Angel 1979).

Several QSO models have invoked an optically thick scattering cloud lying well within the broad-line emission region $\left(R<10^{17} \mathrm{~cm}\right)$ to explain various properties of the optical and X-ray continuum (Katz 1976; Stockman 1978). A disklike optically thin extension of such a cloud could be the origin of the observed polarization. Observationally, this polarization will be difficult to distinguish from polarization arising within 
iNi the central source $\left(R \sim 10^{15} \mathrm{~cm}\right)$. In both cases the $\checkmark$. emission lines will not be polarized and, since the most $\therefore$ likely scatterers are electrons, the polarization should not vary significantly with wavelength. The temporal In behavior of the polarization could be somewhat in different, however, with the scattering cloud producing

$1-1$ constant polarization and the intrinsic polarization perhaps varying somewhat in both degree and angle as in the less active BL Lacertae objects.

In conclusion, we cannot now distinguish galactic or nuclear origins for the polarization and its alignment, but future measurements of emission-line polarization and studies of continuum polarization variability have the potential to do this. In either case, the existence of this polarization and its alignment with the radio lobes may provide valuable information concerning the structure of the central source, the creation of extended radio sources, and the interaction between the central source and the surrounding host galaxy.

This work was begun while two of us (J. R. P. A. and G. K. M.) were guests at the Santa Cruz Summer Workshop on Quasars and Active Galactic Nuclei. We thank Dr. G. Blumenthal for useful discussions. G. K. M. acknowledges a travel grant from the Netherlands Organization for the Advancement of Pure Research (ZWO) and support from the Martin Kellogg Fund. Optical polarimetry at Steward Observatory is supported by NSF grant AST 75-17845.

\section{REFERENCES}

Anderson, B., and Donaldson, W. 1967, M.N.R.A.S., 137, 81.

Angel, J. R. P. 1969, A p. J., 158, 219.

Angel, J. R. P., et al. 1978, Proc. Pittsburgh Conf. BL Lac Objects, ed. A. Wolfe (in press).

Angel, J. R. P., Stockman, H. S., Woolf, N. J., Beaver, E. A., and Martin, P. G. 1976, A p. J.' (Letters), 206, T.5.

Blandford, R. D. 1976, M.N.R.A.S., 176, 465

Conway, R. G., Burn, B. J., and Vallee, J. P. 1977, Astr. Ap. Suppl., 27, 155.

Davis, R. J., Stannard, D., and Conway, R. G. 1977, Nature, 267, 596.

Fanti, C., Fanti, R., Formiggini, L., Lari, C., and Padrielli, L. 1977, Astr. Ap. Suppl., 28, 351.

Fomalont, E. B., and Miley, G. K. 1975, Nature, 257, 99.

Hogg, D. E. $1969, A p . J ., 155,1099$.

Jura, M. 1978, $A p . J ., 223,421$.

Katz, J. I. 1976, Ap. J., 206, 910.

Kinman, T. D. 1977, Nature, 267, 798.

Lovelace, R. V. E. 1976, Nature, 262, 649.

Maza, J., Martin, P. G., and Angel, J. R. P. 1979, in preparation.
Miley, G. K. 1976, Proc. NATO Urbino Summer School, Physics of Radio Sources, ed. G. Setti (Dordrecht: Reidel).

Miley, G. K., and Hartsuijker, A. P. 1978, Astr. Ap. Suppl., 34, 129.

Readhead, A. C. S., Cohen, M. H., and Blandford, R. D. 1978, Nature, 272, 131

Rees, M. 1978, private communication.

Sanders, R. 1974, Nature, 248, 390

Schmidt, G. D., Peterson, B. M., and Beaver, E. A. 1978, Ap. J. (Letters), 220, L31.

Stockman, H. S. 1978, Proc. Pittsburgh Conf. BL Lac Objects, ed. A. Wolfe (in press).

Stockman, H. S., and Angel, J. R. P. 1978, A p. J. (Letters), 220, L67.

Stockman, H. S., Angel, J. R. P., and Beaver, E. A. 1976, Bull. $A A S, 8,495$.

Wardle, J. F. C., and Miley, G. K. 1974, Astr. Ap., 30, 305.

Wilkinson, P. N., Richards, P. J., and Bowden, T. N. 1974. M.N.R.A.S., 168, 515 .

J. R. P. Angel and H. S. Stockman: Steward Observatory, University of Arizona, Tucson, AZ 85721

G. K. Mney: Sterrewacht, Huygens Laboratorium Wassenaarseweg 78, Leiden-2405, The Netherlands 